\title{
Neuere Ergebnisse der Mutterkornalkaloid-Forschung
}

\author{
New Results of Ergot Alkaloid Research
}

\author{
P. A. Stadler \\ Präklinische Forschung Pharma, SANDOZ AG, Switzerland, Basel
}

Received: August 22, 1982

\section{Key Word Index:}

Claviceps purpurea; Fungi; Ergot; New Alkaloids; Biosynthesis; Microbiology.

A review on newly discovered ergot alkaloids, on biosynthetic pathways from tryptophan to the peptide type of alkaloids and on the latest achievements of ergot microbiology is given.

Mutterkorn ist der Menschheit schon seit vielen Jahrhunderten bekannt. Die Droge, offizinell als Secale cornutum bezeichnet, ist das außen dunkelviolett, innen hell gefärbte Dauermycel des Schlauchpilzes Claviceps purpurea (FrIEs) TULASNE, der mit Vorliebe auf reifenden Roggenähren parasitiert.

Schon seit langer Zeit muß Mutterkorn in der Geburtshilfe verwendet worden sein, obwohl der erste schriftliche Hinweis darauf erst in dem im Jahre 1582 erschienenen Kräuterbuch von Adam LoNITZER enthalten ist. In den folgenden Jahrhunderten war die medizinische Verwendung von Mutterkornextrakten umstritten, da die Wirkung der Droge, wie wir heute wissen, wegen der Unbeständigkeit und dem wechselnden Gehalt der Wirkstoffe, oft nicht reproduzierbar war.

Klare Verhältnisse schuf eigentlich erst 1918 die Entdeckung des Ergotamins, 1, des ersten chemisch einheitlichen genuinen Mutterkorn-Peptidalkaloids mit vollem und reproduzierbarem biologischem Wirkungsspektrum [1]. Ergotamin-tartrat wurde in einer ersten Indikation Uteruskontraktion zur Blutstillung in der Nachgeburtsphase in die Therapie eingeführt, welcher später eine zweite, die Migräne-Anfallsbehandlung folgte.

Damit war der Durchbruch erfolgt. Eine intensive, interdisziplinäre Zusammenarbeit von Mikrobiologen, Chemikern, Pharmakologen und Klinikern setzte ein, die zu einer Vielfalt von Therapeutica auf Ergotbasis mit neuen Indikationsgebieten führte.
Einige wesentliche Arbeiten daraus seien hervorgehoben: Im Jahre 1935 erfolgte die gleichzeitige Entdeckung der Ergobasins, 7, durch vier verschiedene Forschungsgruppen [2]. Kurz danach gelangen die ersten Partialsynthesen von Lysergsäure-amiden, darunter die des Ergobasins, 7, 1938 [3], und 1943 die des LSD, 9 [4]. Die katalytische Hydrierung von genuinen Mütterkorn-Peptidalkaloiden führte $1943 \mathrm{zu}$ Dihydro-ergotamin, 2, und zu den 9,10-Dihydro-Derivaten der Alkaloide der Ergotoxingruppe, deren Gemisch unter dem Namen Codergocrine-mesylat therapeutisch genutzt wird [5]. Medizinisches Neuland erschlossen auch die Synthesen des Methysergids, 10, im Jahre 1957 [6] und diejenige von Bromocriptin, 13, synthetisiert 1966 [7] und in die Therapie eingeführt 1976.

Mit den in Figur 1 aufgelisteten Therapeutica haben sich zu den ursprünglichen Indikationsbereichen Uterotonie und Migräne-Anfallsbehandlung die Migräne-Intervallbehandlung, die Venentonisierung, die Hypertonie, die Behandlung des Orthostasesyndroms, der Ausfallserscheinungen des alternden Gehirns, des Parkinsonismus, der Prolactin- und Wachstumshormon bedingten Krankheiten und von bestimmten Hypophysentumoren zugesellt. Es ist abzusehen, daß in Zukunft noch weitere Indikationsgebiete folgen werden.

\section{Seit 1976 entdeckte Alkaloide}

Der erste Schritt auf dem langen Weg zu einem neuen Therapeuticum ist im allgemeinen die Reinherstellung eines Wirkstoffes entweder durch Isolierung aus einer Droge oder durch chemische Synthese. Alle neu isolierten Ergotalkaloide verdienen deshalb spezielles Interesse, weil sie potentielle Therapeutica sind und darüber hinaus auch oft das Wissen über die Biosynthese dieser Verbindungen erweitern. Die bis 1976 aufgefundenen Alkaloide sind in einem Übersichtsartikel aufgelistet [8]. Seitdem aufgefundene Ergotalkaloide sind in Figur 2 zusammengestellt. 

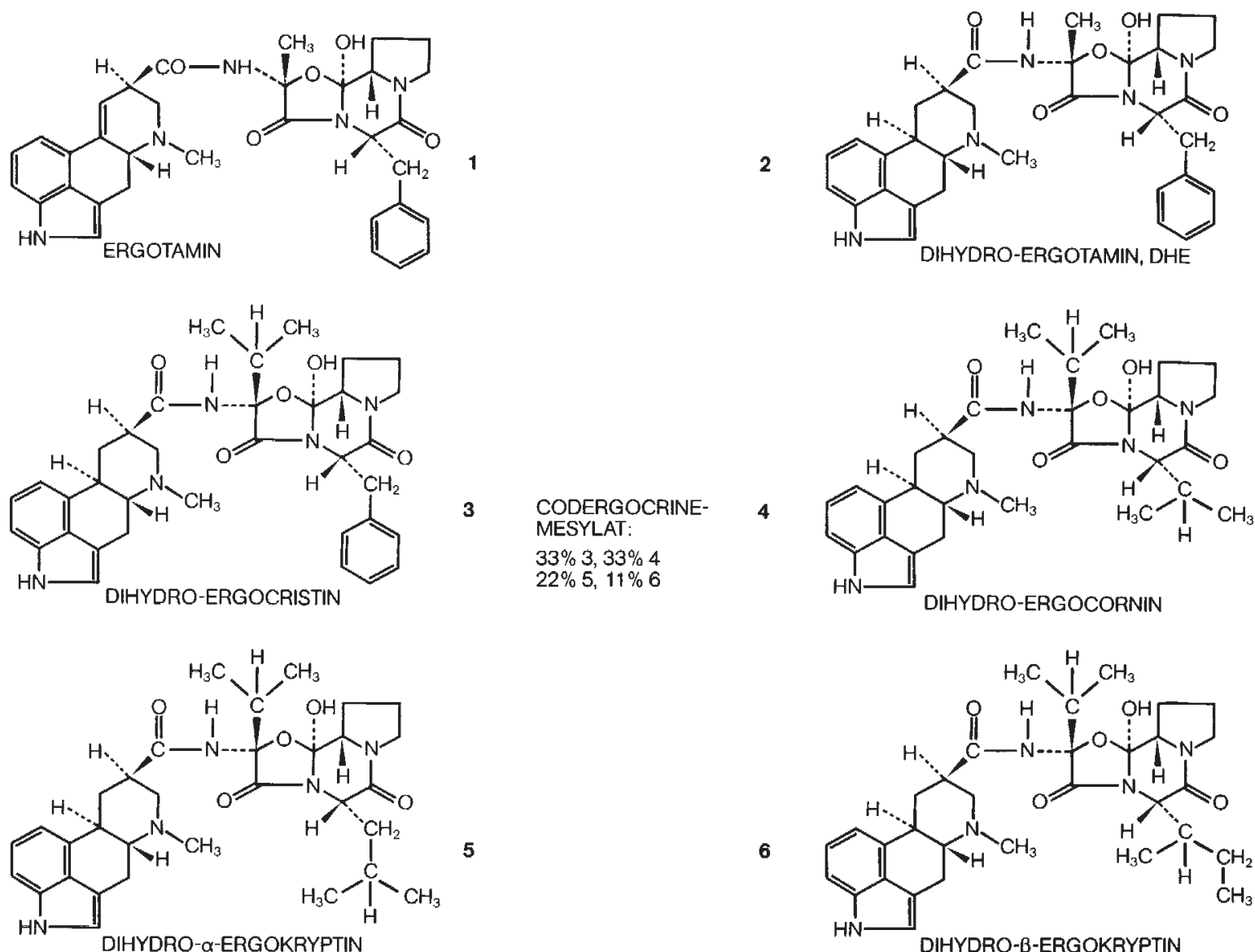

CODERGOCRINE

$33 \% 3,33 \% 4$ $22 \% 5,11 \% 6$

DIHYDRO-ERGOCORNIN

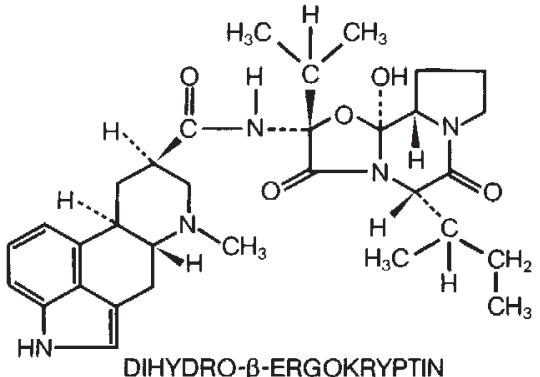

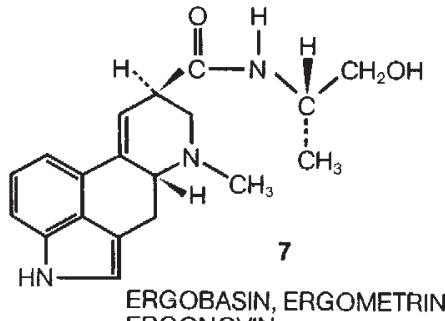<smiles>CCCCNC(=O)OCCN</smiles>

ERGOBASIN, ERGOMETRIN

METHYLERGOBASIN<smiles></smiles>

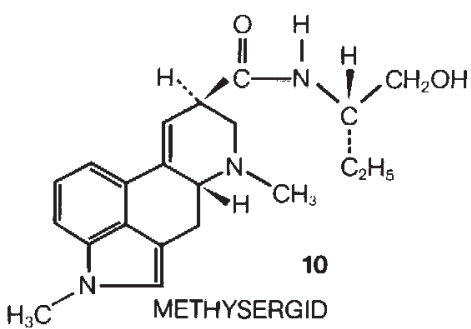

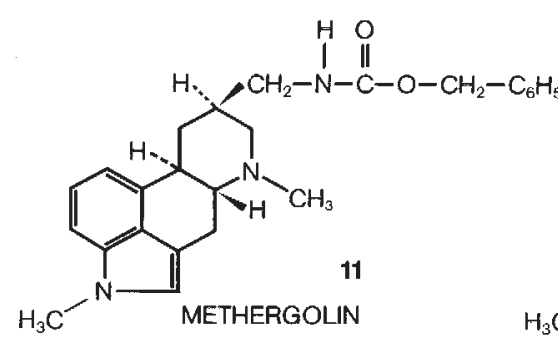<smiles>COC12C[C@H](COC(=O)c3cncc(Br)c3)CN(C)[C@H](Cc3cn(C)c4cccc(c34)C1(O)O)[C@H]2O</smiles>

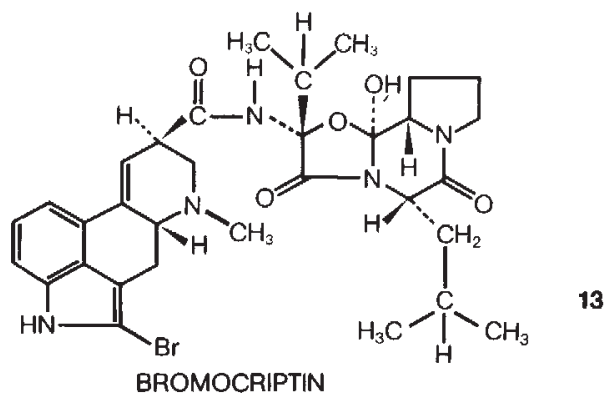<smiles>CCN(C)C(=O)N[C@H]1C=C2c3cccc4[nH]cc(c34)C[C@H]2N(C)C1</smiles>

LISURID

Fig. 1. Therapeutisch genutzte Ergotverbindungen. 
<smiles>CNC1Cc2c[nH]c3cccc(c23)[C@H]1C=C(C)C</smiles>

15<smiles>CN[C@H]1Cc2c[nH]c3cccc(c23)[C@H]1/C=C(\C)C=O</smiles>

16<smiles>CN[C@H]1Cc2c[nH]c3cccc(c23)[C@H]1/C=C(\C)C(=O)O</smiles>

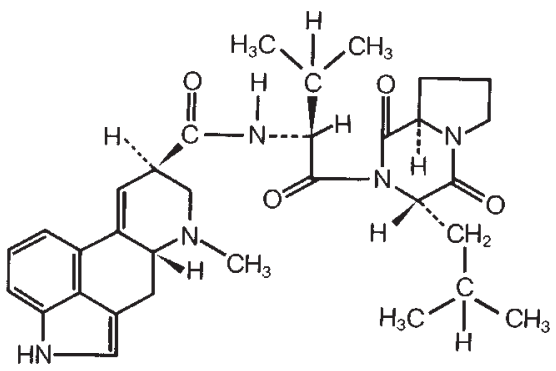

$\alpha$-ERGOKRYPTAM<smiles>C[C@@H]1CN(C)[C@@H]2Cc3c[nH]c4cccc(c34)C2=CCC1=O</smiles>

$\alpha$-ERGOPTIN

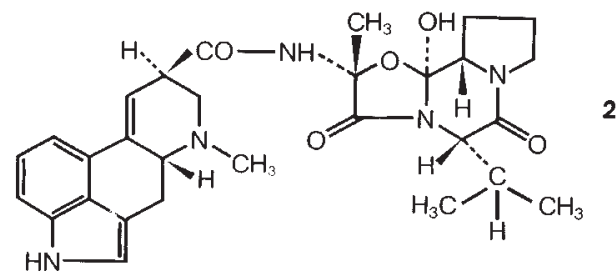

ERGOVALIN

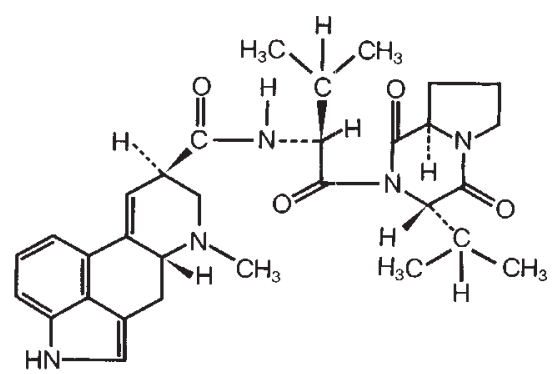

ERGOCORNAM<smiles>C[C@H]1CN(C)[C@@H]2Cc3c[nH]c4cccc(c34)C2=CCC1=O</smiles>

ERGONIN<smiles>CN1CC(O)(C(=O)N[C@@]2(C)C[C@]34CCCN3C(=O)[C@@H](Cc3ccccc3)C4(O)O2)C(=O)N1C</smiles>

8-HYDROXY-ERGOTAMIN
19

21 23

Fig. 2. Seit 1976 neu aufgefundene Ergotalkaloide.

Aus $C$. purpurea, Stamm AA-218, der Agroclavin, 73, und Elymoclavin, 74, als Hauptalkaloide liefert, wurde als neues Nebenalkaloid 6,7-seco-Agroclavin, 15 , isoliert, aus seinen physikalischen Eigenschaften die Struktur abgeleitet und diese durch Partialsynthese aus Agroclavin gesichert [9]. Da das neue Alkaloid 15 nicht in Agroclavin, 73, Elymoclavin, 74, oder Paspalsäure, $\underline{75}$, eingebaut wird, nehmen die
Entdecker an, daß 15 ein durch reduktive Ringöffnung entstandener Metabolit von Agroclavin sei.

Aus einer blockierten Mutante des C. purpurea Stammes Pepty 695/S, der normalerweise hauptsächlich Ergotoxinalkaloide neben größeren Mengen von Ergobasin, 7, und von Clavinalkaloiden produziert, wurde eine morphologisch unveränderte, aber in ihrer Synthesekraft blockierte Mutante Pepty 695/ch 
erhalten, die eine drastisch verringerte Aktivität an Chanoclavin-I-cyclase aufwies und demzufolge anstelle der obengenannten Alkaloide große Mengen an Chanoclavin-I, 72, und eines unbekannten amorphen Alkaloids aufwies. Das neue Alkaloid wurde physikalisch als Chanoclavin-I-aldehyd, 16, identifiziert und seine Struktur durch Reduktion mit $\mathrm{NaBH}_{4}$ zu Chanoclavin-I gesichert [10]. Synthetisches 16 wurde schon früher als Zwischenprodukt der Biosynthese der Ergotalkaloide nachgewiesen [11].

Aus Samen der Convolvulazee Ipomoea violacea, sowie aus dem Schimmelpilz Penicillium islandicum, wurde 1978 ein neues Alkaloid isoliert, als Chanoclavin-I-säure, 17, charakterisiert und in seiner Struktur und Stereochemie durch Partialsynthese aus Chanoclavin-I, 72, gesichert [12]. Damit sind in der Chanoclavin-I-Reihe alle Oxydationsstufen als natürlich vorkommende Alkaloide nachgewiesen.

Nachdem 1973 der erste Vertreter eines grundlegend neuen Typs von Mutterkorn-Peptidalkaloiden, das $\mathrm{N}$-[N-(d-Lysergoyl-L-valyl]-L-phenylalanyl-Dprolin-lactam, 60, heute Ergocristam genannt, isoliert worden war [13], war die Entdeckung weiterer Vertreter dieser am besten Ergopeptame genannten Gruppe nur eine Frage der Zeit. 1981 erfolgte denn auch die Isolierung von $\alpha$-Ergokryptam, 18 , und von Ergocornam, 19, und deren Charakterisierung aus tschechischem $\bar{M}$ utterkorn eines hauptsächlich Ergokryptin und Ergocornin produzierenden C. purpurea Stammes. Bei der Behandlung der drei Alkaloide mit einem Essigester/Wasser/Essigsäure-Gemisch wurden erstmals auch die entsprechenden Verbindungen der d-Isolysergsäure, genannt Ergocristinam, 61, $\alpha$-Ergokryptinam, 62, und Ergocorninam, 65, erhalten [14].

Im Zuge von systematischen analytischen Arbeiten nach Nebenalkaloiden in der Gruppe der eigentlichen Mutterkorn-Peptidalkaloide vom Cyclolty- pus, besser und kürzer Ergopeptine genannt [15], sind letzthin drei neue Alkaloidpaare isoliert worden [16].

Ergostin, 30, der erste Vertreter der Ergoxingruppe, war seinerzeit als Nebenalkaloid des Ergotamins aus Mutterlaugen eines Ergotaminproduktionsstammes isoliert worden [17]. Es lag also nahe, ebensolche Mutterlaugen der Ergokryptin/Ergocorninproduktion nach neuen Alkaloiden der Ergoxingruppe abzusuchen. Natürlich erleichterten synthetisches $\alpha$ Ergoptin und Ergonin die chromatographischen Sucharbeiten beträchtlich [18].

Sowohl für die Isolierung von $\alpha$-Ergoptin, 20, als auch für die von Ergonin, 21, standen Mutterlaugen mit einem chromatographisch ermittelten Gehalt von je etwa $10 \%$ des gesuchten Alkaloids zur Verfügung. Die Reindarstellung der beiden neuen Alkaloide erfolgte in bekannter Weise durch Chromatographien und Kristallisationen der freien Basen als auch ihrer Salze mit di-para-Toluyl-weinsäure. Einer Schätzung nach erreicht der Gehalt von $\alpha$-Ergoptin, 20 , etwa $0,5 \%$ des Gesamtalkaloidgehaltes, der von Ergonin, 21, nur 0,1\%.

Für die Isolierung von Ergovalin, 22, standen synthetisches Vergleichsmaterial [18] und der Experimentalstamm C. purpurea 235 zur Verfügung, in dessen Rohalkaloidgemisch neben sehr viel $\alpha$-Ergosin etwa $4 \%$ Ergovalin chromatographisch nachweisbar war. Die Isolierung von 22 gelang mit Hilfe eines speziellen chromatographischen Systems. Vor kurzer Zeit wurden Ergovalin, 22, Ergovalinin, 29, dessen Paarling und 6,7-seco-Agroclavin, 15, auch in Kulturen von Epichloe typhina nachgewiesen [19].

Aus einem hauptsächlich Ergotamin produzierenden C. purpurea Stamm wurde 1979 ein neues Mutterkorn-Peptidalkaloid isoliert, das überraschenderweise die übliche Isomerisierungsreaktion zur entsprechenden Isoform nicht zeigte [20]. Diese Beob-

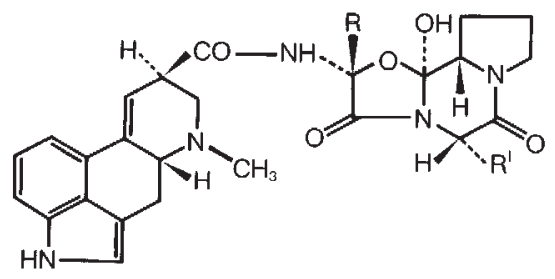

\begin{tabular}{|c|c|c|c|}
\hline$R^{1}$ & $\begin{array}{l}\text { Ergotamin- } \\
\text { gruppe } \\
\mathrm{R}=\mathrm{CH}_{3}\end{array}$ & $\begin{array}{l}\text { Ergoxin- } \\
\text { gruppe } \\
\mathrm{R}=\mathrm{C}_{2} \mathrm{H}_{5}\end{array}$ & $\begin{array}{l}\text { Ergotoxin- } \\
\text { gruppe } \\
\mathrm{R}=\mathrm{CH}\left(\mathrm{CH}_{3}\right)_{2}\end{array}$ \\
\hline $\mathrm{CH}_{2}-\mathrm{C}_{6} \mathrm{H}_{5}$ & $\begin{array}{l}\text { ERGOTAMIN, } 1 \\
\text { ERGOTAMININ, } 24\end{array}$ & $\begin{array}{l}\text { ERGOSTIN, } 30 \\
\text { ERGOSTININ, } 31\end{array}$ & $\begin{array}{l}\text { ERGOCRISTIN, } 36 \\
\text { ERGOCRISTININ, } 37\end{array}$ \\
\hline $\mathrm{CH}_{2}-\mathrm{CH}\left(\mathrm{CH}_{3}\right)_{2}$ & $\begin{array}{l}\alpha \text {-ERGOSIN, } 25 \\
\alpha \text {-ERGOSININ, } 26\end{array}$ & $\begin{array}{l}\alpha \text {-ERGOPTIN, } 20 \\
\alpha \text {-ERGOPTININ, } 32\end{array}$ & $\begin{array}{l}\alpha \text {-ERGOKRYPTIN, } 38 \\
\alpha \text {-ERGOKRYPTININ, } 39\end{array}$ \\
\hline $\mathrm{CHCH}_{3}-\mathrm{C}_{2} \mathrm{H}_{5}$ & $\begin{array}{l}\beta \text {-Ergosin, } 27 \\
\beta \text {-Ergosinin, } 28\end{array}$ & $\begin{array}{l}\beta \text {-Ergoptin, } 33 \\
\beta \text {-Ergoptinin, } 34\end{array}$ & $\begin{array}{l}\beta \text {-ERGOKRYPTIN, } 40 \\
\beta \text {-ERGOKRYPTININ, } 41\end{array}$ \\
\hline $\mathrm{CH}\left(\mathrm{CH}_{3}\right)_{2}$ & $\begin{array}{l}\text { ERGOVALIN, } 22 \\
\text { ERGOVALININ, } 29\end{array}$ & $\begin{array}{l}\text { ERGONIN, } 21 \\
\text { ERGONININ, } 35\end{array}$ & $\begin{array}{l}\text { ERGOCORNIN, } 42 \\
\text { ERGOCORNININ, } 43\end{array}$ \\
\hline
\end{tabular}


achtung sowie eine genaue NMR-Analyse führte zur Struktur des 8-Hydroxy-ergotamins, 23. In 23 bleibt die absolute Konfiguration in Stellung 8 noch abzuklären, doch deutet der gemessene, niedrige optische Drehungswert von 23 in Pyridin darauf hin, daß das $8 \alpha$-Hydroxy-ergotamin vorliegt. Im Moment ist noch nicht abzuschätzen, ob 23 als erster Vertreter einer neuen, genuinen Ergotalkaloidgruppe einzustufen ist oder ob es sich um einen Metaboliten des Ergotamins handelt.

Abschließend $z u$ diesem Kapitel einige Bemerkungen zur Anzahl und Systematik der Ergotalkaloide. Eine erste Gruppe, die der Clavine, umfaßt heute knapp 40 tri- und tetrazyklische Alkaloide, die zweite Gruppe, die der einfachen Lysergsäurederivate, nur Paspalsäure, Lysergsäure/Isolysergsäure, deren primäre Amide und die beiden Hydroxyäthylamide sowie den Paarling Ergobasin/Ergobasinin. Von den therapeutisch wichtigen Ergopeptinen kennt man heute 10 Paarlinge, die sich zwanglos in drei natürliche Gruppen einteilen lassen.

Während allen Ergopeptinen L-Prolin als Baustein gemeinsam ist, enthalten die Mitglieder einer Gruppe darüber hinaus noch die gleiche $\alpha$-Hydroxy$\alpha$-amino-carbonsäure, was formelmäßig einem identischen Substituenten R in Stellung 2' entspricht. Die Mitglieder einer Gruppe unterscheiden sich nur in der Struktur der zweiten Aminosäure, also im Substituenten $\mathrm{R}^{\prime}$. Da Claviceps als zweite, variable Aminosäure merkwürdigerweise nur L-Valin, LLeucin, L-Isoleucin und L-Phenylalanin in den Peptidteil einbaut, umfaßt eine Alkaloidgruppe maximal vier Paarlinge. Das ist der Fall bei der Ergotoxingruppe. Die Zahl der bisher bekannten, vom Pilz in den Peptidteil eingebauten $\alpha$-Hydroxy- $\alpha$-amino-carbonsäuren beschränkt die Zahl der Alkaloidgruppen auf drei. Mit der Identifikation von Ergovalin, 22, $\alpha$ Ergoptin, 20 und Ergonin, 21, als natürlich vorkom- mende Alkaloide konnten in Schema 3 wichtige Löcher gestopft werden, so daß die Ergotamin- und die Ergoxingruppe heute je drei natürliche Paarlinge enthalten. Leider sind bis heute die beiden Paarlinge $\beta$-Ergosin, 27, $\beta$-Ergosinin, 28, und $\beta$-Ergoptin, 33, $\beta$-Ergoptinin, 34, nicht als natürliche Alkaloide nachgewiesen, sie werden deshalb im Schema klein geschrieben.

Im Sinne eines zur Zeit noch stark hypothetisch geprägten Ausblicks in die Zukunft sei es erlaubt, für die Gruppe der Ergopeptame ein der natürlichen Gruppeneinteilung der Ergopeptine völlig parallel laufendes Schema aufzustellen. Denn es erscheint plausibel, daß jedes in der Natur vorkommende Ergopeptin von einem entsprechenden Ergopeptam begleitet wird, weil diese ja allem Anschein nach entweder eine Sackgasse oder einen zweiten Endpunkt der Biosynthese der Mutterkorn-Peptidalkaloide darstellen. Leider ist in diesem Schema 4 zur Zeit die Zahl der noch unbekannten und deshalb klein geschriebenen Alkaloide wesentlich größer als bei den Ergopeptinen.

\section{Zur Biosynthese der Ergotalkaloide}

Die Abklärung der Biosynthese der Ergotalkaloide ist in den letzten zwanzig Jahren zu einem zentralen Thema der Mutterkornforschung geworden. Wegen der Komplexizität dieses Problemkreises ist eine in die Details gehende Behandlung im Rahmen dieses Artikels unmöglich und auch unnötig, bestehen doch bereits verschiedene Übersichtsartikel darüber $[8,21,49,50]$. Die molekularen Bausteine und die gesicherten, wichtigen Zwischenstufen der Biosynthese der Lysergsäure sollen jedoch kurz aufgezeigt werden, da sie für das Verständnis des mikrobiologischen Teils der Ergotforschung nötig sind. Uber die sich daran anschließende Biosynthese der Ergopepti-
Fig. 4. Hypothetische Gruppen der Ergopeptame.

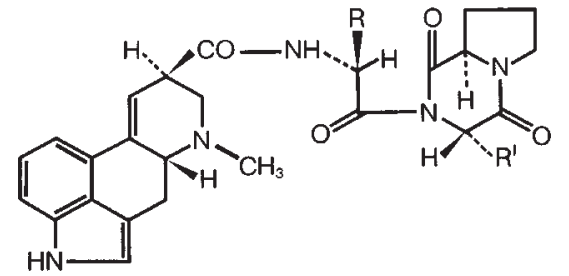

\begin{tabular}{|l|l|l|l|}
\hline & \multicolumn{1}{|c|}{$\begin{array}{l}\text { Ergotamam- } \\
\text { gruppe } \\
\mathrm{R}=\mathrm{CH}_{3}\end{array}$} & $\begin{array}{l}\text { Ergoxam- } \\
\text { gruppe } \\
\mathrm{R}=\mathrm{C}_{2} \mathrm{H}_{5}\end{array}$ & $\begin{array}{l}\text { Ergotoxam- } \\
\text { gruppe } \\
\mathrm{R}=\mathrm{CH}\left(\mathrm{CH}_{3}\right)_{2}\end{array}$ \\
\hline $\mathrm{CH}_{2}-\mathrm{C}_{6} \mathrm{H}_{5}$ & $\begin{array}{l}\text { Ergotamam, 44 } \\
\text { Ergotaminam, 45 }\end{array}$ & $\begin{array}{l}\text { Ergostam, 52 } \\
\text { Ergostinam, 53 }\end{array}$ & $\begin{array}{l}\text { ERGOCRISTAM, 60 } \\
\text { ERGOCRISTINAM, 61 }\end{array}$ \\
\hline $\mathrm{CH}_{2}-\mathrm{CH}\left(\mathrm{CH}_{3}\right)_{2}$ & $\begin{array}{l}\alpha \text {-Ergosam, 46 } \\
\alpha \text {-Ergosinam, 47 }\end{array}$ & $\begin{array}{l}\alpha \text {-Ergoptam, 54 } \\
\alpha \text {-Ergoptinam, 55 }\end{array}$ & $\begin{array}{l}\alpha \text {-ERGOKRYPTAM, 18 } \\
\alpha \text {-ERGOKRYPTINAM, 62 }\end{array}$ \\
\hline $\mathrm{CHCH}_{3}-\mathrm{C}_{2} \mathrm{H}_{5}$ & $\begin{array}{l}\beta \text {-Ergosam, 48 } \\
\beta \text {-Ergosinam, 49 }\end{array}$ & $\begin{array}{l}\beta \text {-Ergoptam, 56 } \\
\beta \text {-Ergoptinam, 57 }\end{array}$ & $\begin{array}{l}\beta \text {-Ergokryptam, 63 } \\
\beta \text {-Ergokryptinam, 64 }\end{array}$ \\
\hline $\mathrm{CH}\left(\mathrm{CH}_{3}\right)_{2}$ & $\begin{array}{l}\text { Ergovalam, 50 } \\
\text { Ergovalinam, 51 }\end{array}$ & $\begin{array}{l}\text { Ergonam, 58 } \\
\text { Ergoninam, 59 }\end{array}$ & $\begin{array}{l}\text { ERGOCORNAM, 19 } \\
\text { ERGOCORNINAM, 65 }\end{array}$ \\
\hline
\end{tabular}




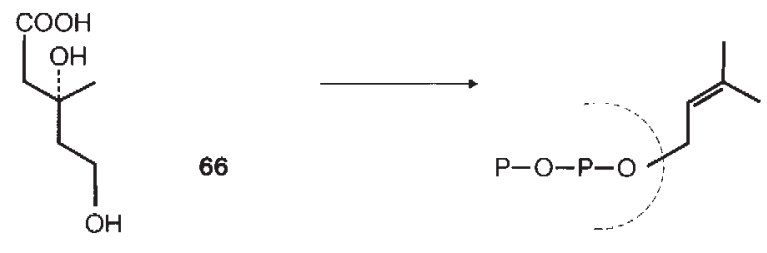

67

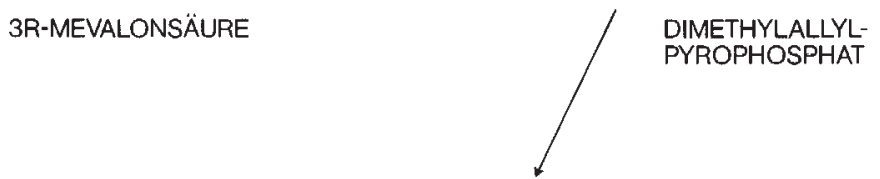

d-LYSERGSÄURE

68
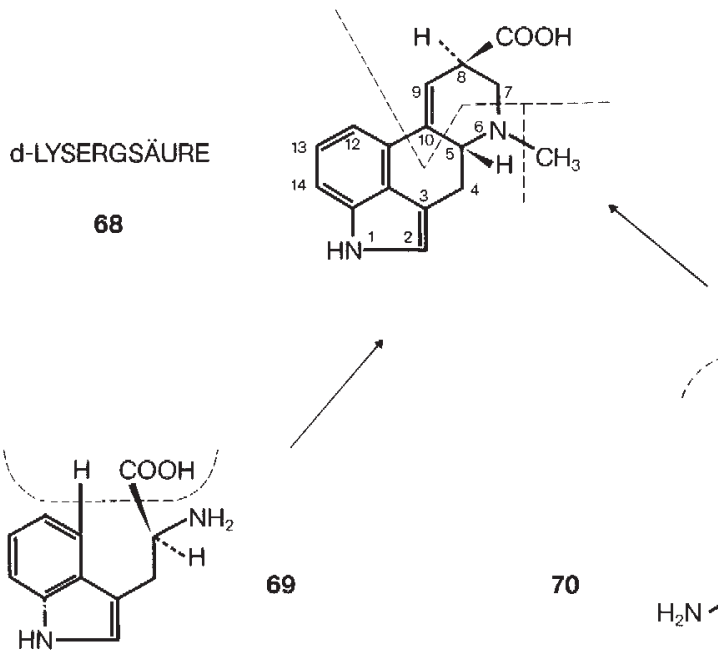

69

70

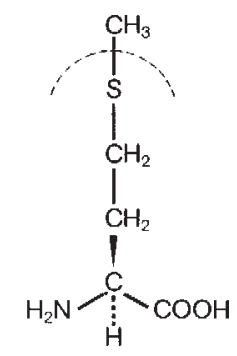

L-TRYPTOPHAN

L-METHIONIN

Fig. 5. Biosynthetische Ursprünge des Ringsystems der d-Lysergsäure.

ne sei, da sie eigene Arbeiten betrifft, etwas ausführlicher berichtet.

In Figur 5 sind die in jeder lebenden Zelle vorkommenden chemischen Bausteine zusammengestellt, aus welchen $C$, purpurea die d-Lysergsäure, 68 , synthetisiert. Fütterungsexperimente mit verschiedenartig markiertem L-Tryptophan, 69 , unter wechselnden Bedingungen an diversen Pilzstämmen führten zu hohen Einbauraten, so daß an der Precursorfunktion von 69 heute nicht mehr zu zweifeln ist $[22,23]$. Allerdings gehen dabei die Carboxylgruppe und das Wasserstoffatom in Stellung 4 verloren. Bemerkenswert ist ferner der Konfigurationswechsel in Stellung $5 \mathrm{im}$ Verlaufe der Biosynthese von $69 \mathrm{zu} 68$. Als zweiter, wesentlicher Baustein für das Ringsystem der dLysergsäure erwies sich die 3R-Mevalonsäure, 66, die zunächst, unter Verlust ihrer Carboxylgruppe, in Dimethylallylpyrophosphat, 67, umgewandelt wird. Der Einbau von 67 in das L-Tryptophan, 69 , erfolgt in Stellung 4 des Indolgerüstes, wobei das sich hier befindende Wasserstoffatom verloren geht $[23,24]$.
Der Beitrag von L-Methionin, 70, an das Lysergsäuremolekül ist, wie Einbauversuche mit der markierten Aminosäure gezeigt haben, die S-Methylgruppe [25]. Diese Methylgruppe alkyliert, allem Anschein nach in intakter Form, die primäre Aminogruppe einer noch $\mathrm{zu}$ bestimmenden Stufe der Biosynthese zwischen dem Dimethylallyl-L-tryptophan, 71, und dem Chanoclavin-I, 72, sie findet sich in hoher Einbaurate schießlich am Stickstoffatom in Stellung 6 der d-Lysergsäure wieder. Auch markiertes Formiat wurde mit geringerer Einbaurate in die gleiche Stelle eingebaut.

4-Dimethylallyl-L-tryptophan, 71, ist als Zwischenprodukt der Biosynthese aus Ergotkulturen isoliert worden, in denen der weitere Verlauf des enzymatischen Geschehens durch Sauerstoffausschluß [26] oder aber durch Zugabe von Ethionin [27] verhindert worden war. Ferner konnte ein Enzym isoliert werden, welches 71 in vitro aus 67 und 69 synthetisiert. Zusammen mit der hohen Einbaurate in Lysergsäurederivate, welche markiertes 71 in Kulturen von $C$. paspali aufweist [28], lassen diese Beobachtungen kaum einen Zweifel übrig, daß Dimethylallyl-L-tryptophan, 71, der erste Schritt der Biosynthese der d-Lysergsäure, 68 , darstellt.

Chanoclavin-I, 72, ein schon längere Zeit bekanntes Clavinalkaloid [29], stellt den nächsten Fixpunkt der Biosynthese der d-Lysergsäure dar [30 bis 34]. Zum Übergang von 71 in 72 ist zu bemerken, daß die in Formel 71 mit einem Stern markierte und zur Doppelbindung in trans-Lage stehende Methylgruppe in Verbindung 72 in der cis-Position aufgefunden wurde; es muß also in der biosynthetischen Stufenfolge zwischen dem 4-Dimethylallyl-L-tryptophan und dem Chanoclavin-I eine Isomerisierung der Doppelbindung stattgefunden haben [35].

Chanoclavin-I-aldehyd, 16 , ist als neu aufgefundenes Clavinalkaloid schon erwähnt worden [10]. Die Rolle von 16 als Zwischenstufe der Biosynthese der d-Lysergsäure, 68 , ist schon früh aufgezeigt worden [11], da markiertes Chanoclavin-I-aldehyd, 16, spezifisch in Elymoclavin, 74, eingebaut wird. Aber es scheint, daß sich hier die Biosynthese in verschiedene Richtungen hin verzweigt. Obwohl auch in einem zellfreien System (erhalten aus Claviceps-Mycelien) aus markiertem Chanoclavin-I, 72, nur Elymoclavin, 74, aber kein Agroclavin, 73 erhalten worden ist [36],

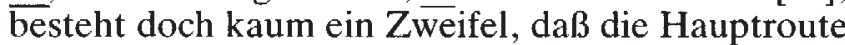
der Biosynthese von 16 über Agroclavin, 73, zu Elymoclavin, 74 , führt. $\overline{A b} g e s e h e n$ davon, da $\bar{B}$ Agroclavin neben Elymoclavin, beispielsweise im Mutterkorn von Pennisetum typhoideum Rich. als Hauptalkaloid, also als Endpunkt der Biosynthese, auftritt [37], ist die Sequenz Chanoclavin-I $\rightarrow$ Agroclavin$\rightarrow$ Elymoclavin gut bewiesen $[30,38]$. Bei diesem Übergang muß eine zweite Isomerisierung der Doppelbindung eingetreten sein, denn die markierte, mit einem Sternchen bezeichnete und cis-ständige $\mathrm{Me}$ - 

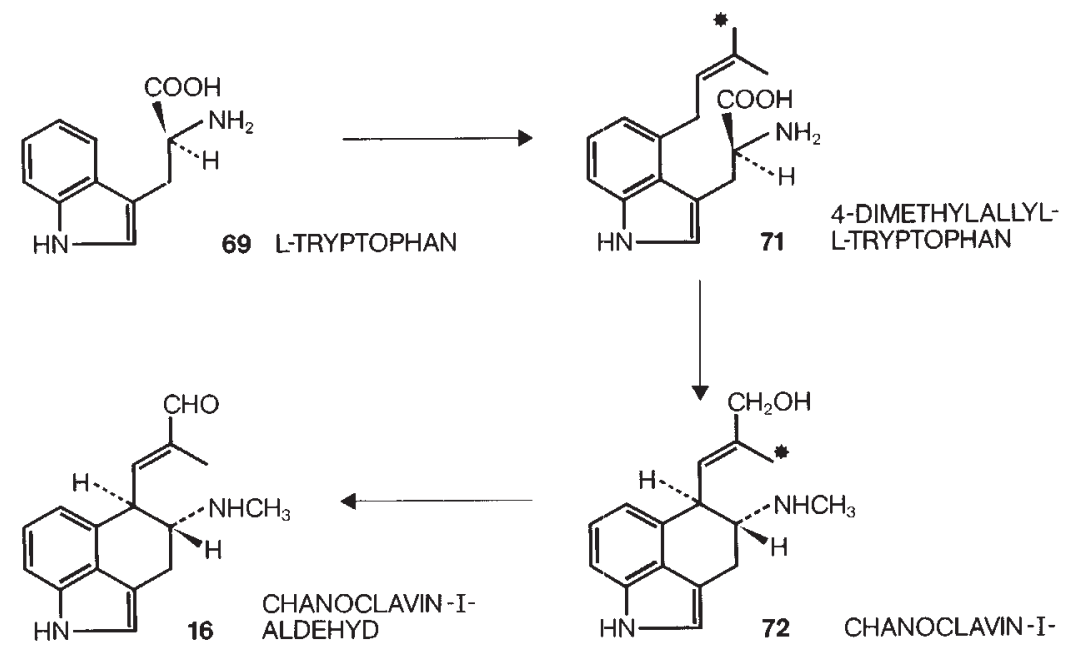

Fig. 6. Gesicherte Zwischenstufen der Biosynthese der d-Lysergsäure.
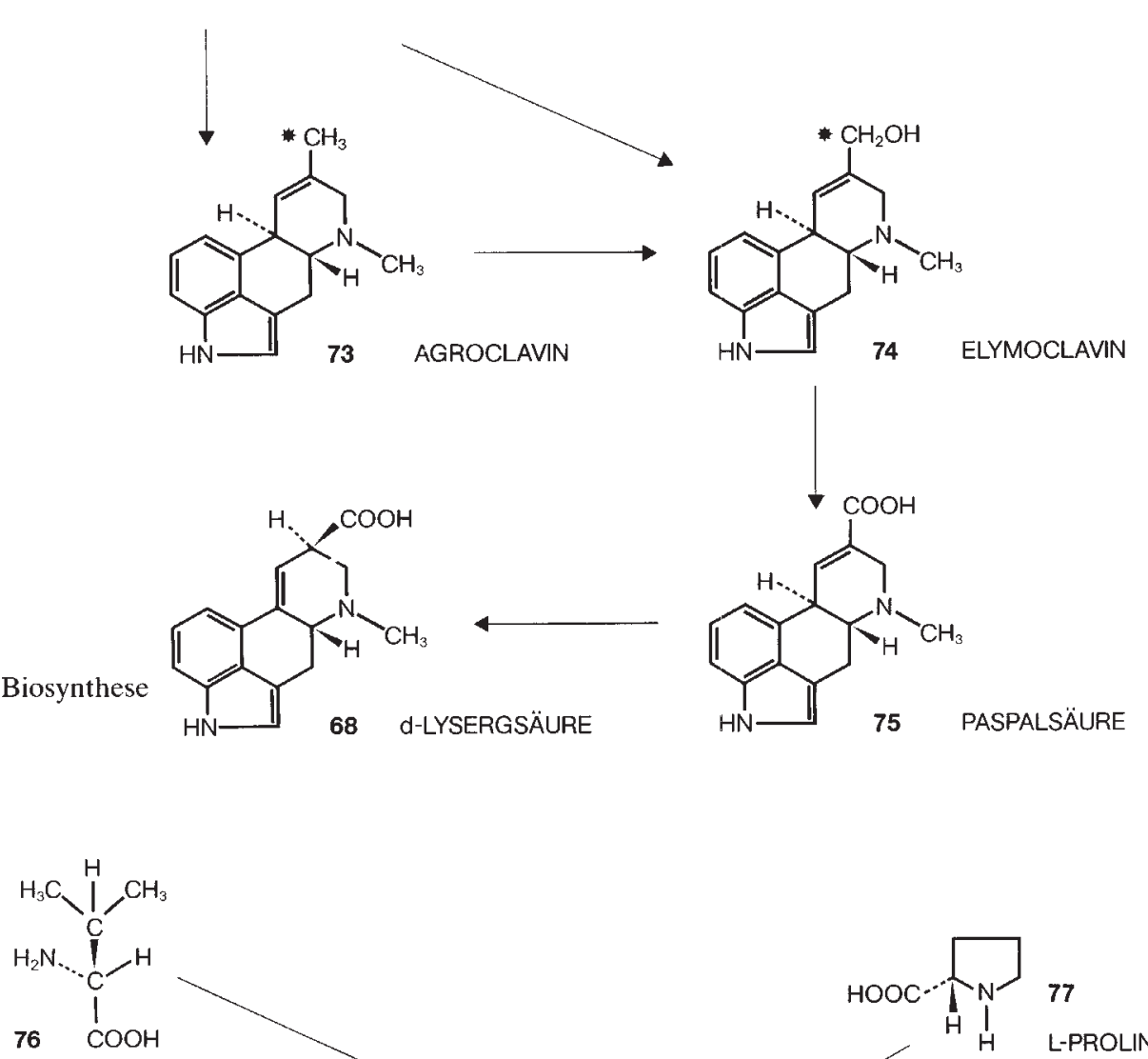

Fig. 7. Biosynthetische Bausteine der Ergopeptine, dargestellt am Beispiel des Ergocristins.

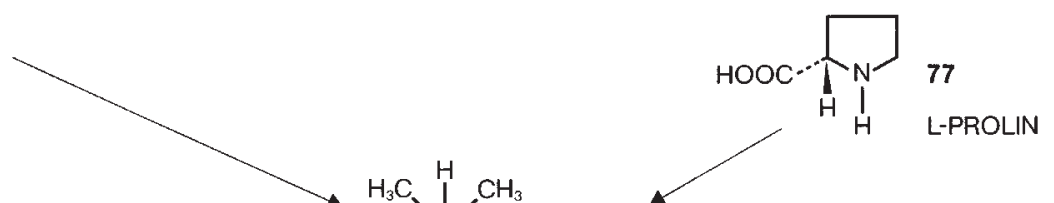

L-VALIN<smiles>CN(C)C(=O)[C@H]1C=C2c3cccc4[nH]cc(c34)C[C@H]2N(C)C1</smiles><smiles>CCOC(=O)OCC</smiles>

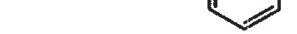

36

ERGOCRISTIN<smiles>[3H]C[123I]</smiles><smiles>N[C@H](Cc1ccccc1)C(=O)O</smiles> 
thylgruppe von Chanoclavin-I, 72, findet sich in Stellung 8 von Agroclavin, 73, beziehungsweise als $\mathrm{Hy}-$ droxymethylgruppe von Elymoclavin, 74, wieder $[31,32]$.

Der biosynthetische, oxydative Übergang von Elymoclavin, 74, in verschiedene Derivate der Lysergsäure wurde schon 1962 aufgezeigt [39]. Zusätzlich erscheint bemerkenswert, daß ein natürlich vorkommender Stamm von $C$. paspali Stevens et Hall die Paspalsäure, 75, als Hauptalkaloid synthetisiert [40]. Allem Anschein nach muß Paspalsäure als Precursor von d-Lysergsäure angesehen werden, denn in markierter Form wurde sie mit guter Einbaurate in Lysergsäureamide eingebaut [41].

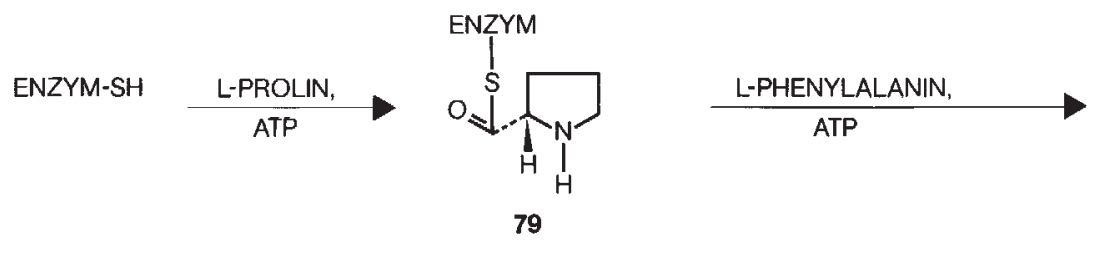

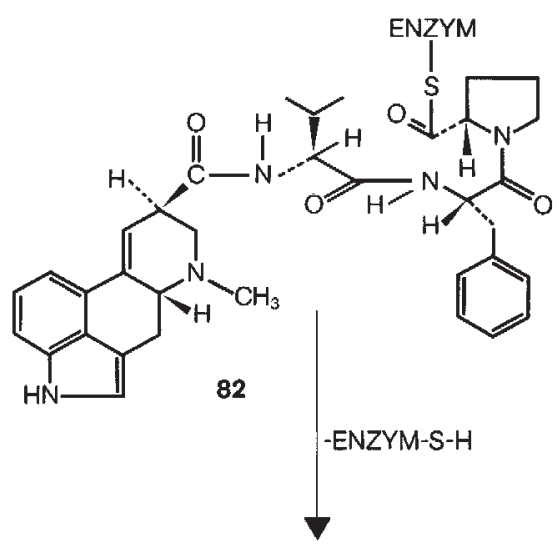

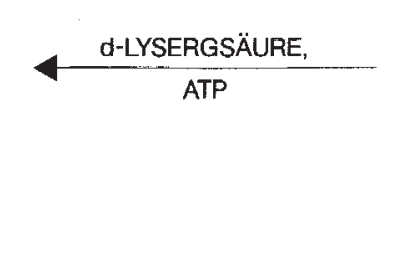

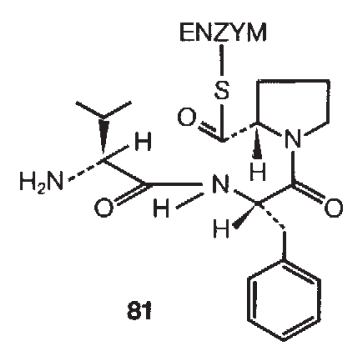

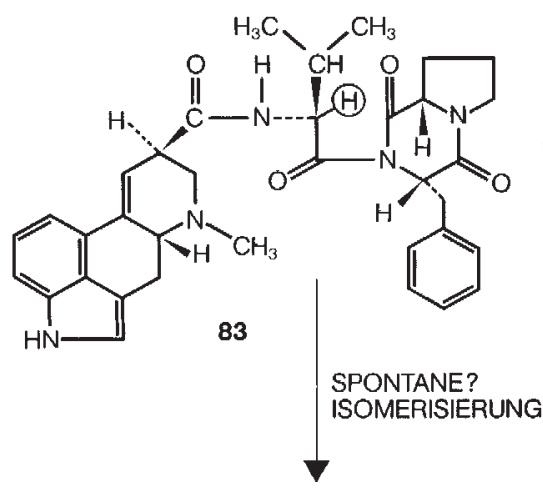

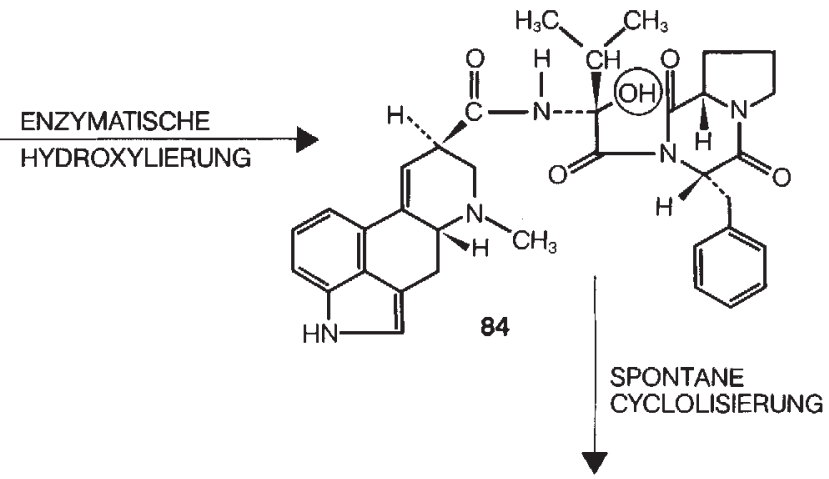<smiles>CC(C)[C@@H](C(=O)N1C(=O)[C@@H]2CCCN2C1=O)N1CC=C2c3cccc4[nH]cc(c34)C[C@H]2N(C)C[C@@H](C(C)C)C1=O</smiles>

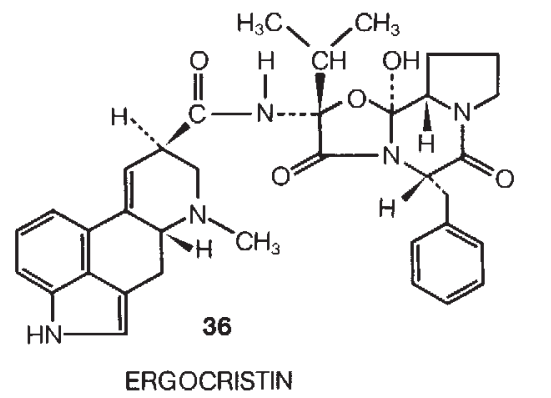

Fig. 8. Hypothetisches Biosyntheseschema der Ergopeptine und Ergopeptame, dargestellt am Beispiel von Ergocristin und Ergocristam. 
Der weitere Verlauf der Biosynthese von der d-Lysergsäure, 68, zu den Ergopeptinen und Ergopeptamen soll am Beispiel des Ergocristins erläutert werden, da sowohl Ergocristin, 36, [42] als auch Ergocristam, 60, [13] die ersten isolierten Alkaloide ihrer Gruppen waren und wesentliche Arbeiten zur Abklärung der Biosynthese an diesen Alkaloiden ausgeführt worden sind.

In Figur 7 sind die heute gut gesicherten Bausteine von Ergocristin, 36, als Beispiel eines Ergopeptins zusammengestellt. Wie erwartet sind dies die d-Lysergsäure, 68, [43, 44], L-Valin, 76, [44, 45], als Precursor für die $\alpha$-Hydroxy- $\alpha$-amino-carbonsäure (es könnte für andere Ergopeptine auch L- $\alpha$-Aminobuttersäure oder L-Alanin sein), L-Prolin, 77, [46, 47], als gemeinsamer Baustein aller Peptidalkaloide und L-Phenylalanin, 78, [47, 48]. (Die letztgenannte Aminosäure ist in andern Ergopeptinen durch L-Valin, L-Leucin oder L-Isoleucin ersetzt). Nachdem die obengenannten Precursormoleküle für die Ergopeptine durch Einbauversuche mit den entsprechenden markierten Verbindungen abgeklärt waren, stellte sich die fundamentale Frage, wie diese Bausteine zu den Ergopeptinen zusammentreten.

Im Laufe der letzten 15 Jahre sind einige Schemata für die Biosynthese der Ergopeptine zur Diskussion gestellt worden. Eine erste Hypothese, 1966 postuliert [49], ist heute nicht mehr aktuell, da sie sich auf N-Lysergoyl-L-valin-methylester als Zwischenprodukt stützte, eine Verbindung, die inzwischen als Artefakt erkannt ist [13]. Eine zweite, 1968 umrißmäßig und rein hypothetisch vorgeschlagen [50], wurde seither, vor allem vom Autor dieses Artikels, weiter entwickelt und durch Forschungsresultate gestützt $[13,51,52]$.

Es sind um 1970 herum viele Fütterungsversuche mit radioaktiv markierten $\mathrm{N}$-Lysergoyl-aminosäuren, mit Dipeptiden, Diketopiperazinen und auch mit Tripeptiden an Claviceps-Stämmen durchgeführt worden [8]. Die Einbauraten in den biosynthetisierten Ergopeptinen deuteten unterschiedslos darauf hin, daß alle diese Verbindungen erst nach einem Abbau zu den einzelnen Aminosäuren in die Ergopeptine eingebaut wurden [8]. Ein wertvolles Ergebnis zeitigten diese an sich negativ verlaufenen Versuche trotzdem: Immer, wenn radioaktiv markiertes Valin an Ergocornin produzierende Stämme verabreicht wurde, beobachtete man im isolierten Ergocornin eine höhere spezifische Aktivität des $\alpha$-Hydroxy-L-valin-Bausteins im Vergleich zu der des daraus ebenfalls angefallenen L-Valins [44, 45]. Die Interpretation dieser Beobachtungen führte zum Schluß, daß die Peptidkette der Ergopeptine vom Prolin-Ende her aufgebaut wird [45] und daß dieser Aufbau konzertiert an einem Multi-Enzym-Komplex erfolgt [51].

Nach dem üblichen Schema der Peptidbildung würde also das an der Carboxylgruppe (wahrschein- lich über ein Schwefelatom) an ein Enzym fixierte LProlin, 79, über die Stufen 80 und 81 in das lineare Lysergoyl-tripeptid 82 übergeführt.

Von rein chemischen Experimenten her ist bekannt, daß zu 82 analoge Tripeptide, deren C-terminale L-Prolin-carboxylgruppe in Form eines aktivierten Esters vorliegt, mit Leichtigkeit je nach Struktur zu N-Acyldioxopiperazinen und sogar zu Cyclolen cyclisieren [53, 54]. Aktiviertes C-terminales Prolin ist für solche Cyclisationen eine Conditio sine qua non [54].

In völliger Analogie zu den angedeuteten chemischen Reaktionsverläufen wäre als nächste Stufe der Biosynthese die Cyclisation des Lysergoyl-tripeptids 82 zur noch unbekannten Schlüsselverbindung $83 \mathrm{zu}$ postulieren, in welcher sich der Biosynthese zwei Wege öffnen:

Aus dem Verhalten von analogen Verbindungen her ist bekannt [55], daß sich das $\alpha$-Wasserstoffatom des L-Prolinrings von 83 in einer energetisch instabilen Lage befindet und mit Leichtigkeit in die entsprechende Verbindung mit einem Prolinring in D-Konfiguration, also in Verbindung 60 , übergehen kann. Verbindung 60 ist aber identisch mit Ergocristam, welches 1973 als erster Vertreter der Ergopeptame aus dem Mycel eines hauptsächlich Ergocristin produzierenden $C$. purpurea Stammes isoliert worden ist [13]. Damit wäre ein erster Endpunkt der Biosynthese der Ergot-Peptidalkaloide erreicht.

Wenn hingegen in der Schlüsselverbindung 83 das $\alpha$-Wasserstoffatom der ersten Aminosäure, also des Valins, enzymatisch zur Hydroxylgruppe oxydiert wird, so würde intermediär Verbindung 84 anfallen, die nach unseren Kenntnissen aus der chemischen Ergopeptinsynthese [54, 55] sofort, spontan und stereospezifisch zu Ergocristin, 36, dem zweiten Endpunkt der Biosynthese, cyclolisiert.

Aus dem Dargelegten geht hervor, daß die Biosynthese-Hypothese der Ergopeptine und Ergopeptame von Figur 8 noch nicht in wünschenswertem Ausmaß experimentell gesichert ist. Eine Isolierung der Zwischenprodukte $79,80,81$ und 82 erscheint schwierig, die von 84 unmöglich und die der nach unseren Kenntnissen sehr instabilen Schlüsselverbindung 83 ist bisher nicht gelungen. Die Isolierung von Ergocristam, 60, die die Hypothese eigentlich zum Leben erweckt hat [13], macht zwar die Existenz der Schlüsselverbindung $\underline{83} \mathrm{sehr}$ wahrscheinlich, beweist sie aber nicht.

In Kenntnis dieser Schwierigkeiten wurde nach einem andern Weg gesucht, die dargelegte Biosynthese-Hypothese zu stützen und in einer biomimetischen Totalsynthese der Ergopeptine auch gefunden [52].

Die chemische Synthese wurde so angelegt, daß sie nach Möglichkeit der Biogenese-Hypothese analog verläuft; Unterschiede ergaben sich zwangsläufig aus den beschränkten chemischen Möglichkeiten. So 
BIOSYNTHESE-HYPOTHESE

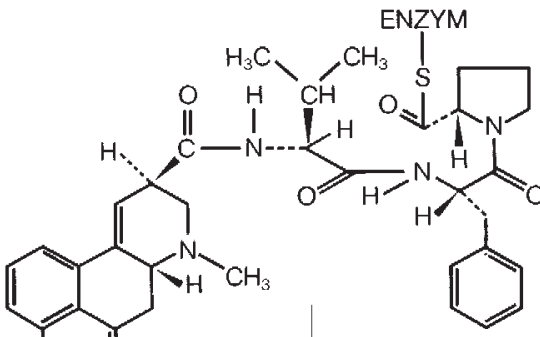

82<smiles>CC(C)[C@H](C(=O)N1C(=O)[C@H]2CCCN2C1=O)N(C)C(=O)[C@@H]1CN(C)[C@@H]2Cc3c[nH]c4cccc(c34)C2=CCC1=O</smiles>

83

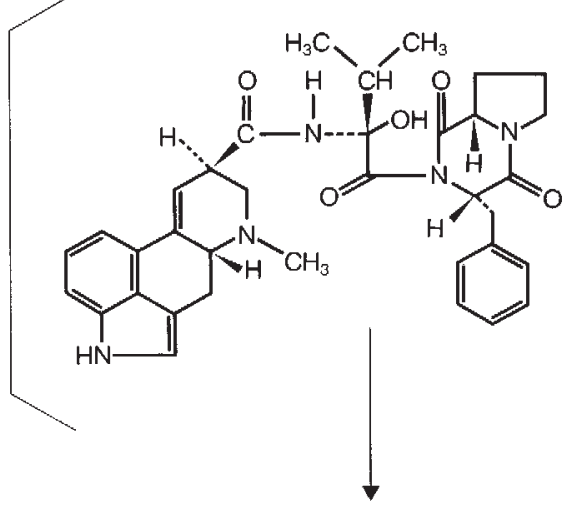

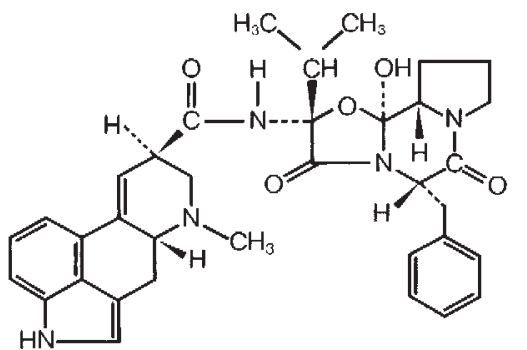

CHEMISCHE, BIOMIMETISCHE SYNTHESE

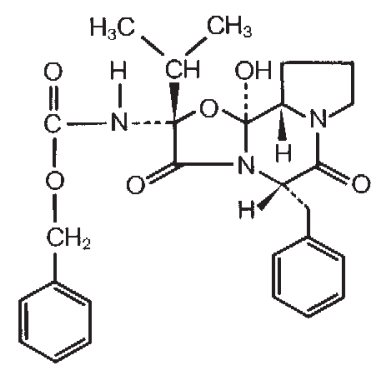

ERGOCRISTIN

Fig. 9. Vergleich relevanter Stufen der Biosynthese-Hypothese mit der zweiten, chemischen Totalsynthese der Ergopeptine.

mußte beispielsweise in vitro an Stelle der oxydationsempfindlichen Lysergsäure mit der N-Benzyloxycarbonyl-Schutzgruppe gearbeitet werden, diese ließ sich übrigens am Ende der Synthese glatt durch
Lysergsäure ersetzen. Auch mußte aus Stabilitätsgründen bei der chemischen Synthese die Folge der beiden relevanten Stufen umgekehrt werden. Die Einführung der $\alpha$-Hydroxy- $\alpha$-amino-gruppe (Stufe 
$\underline{85} \rightarrow$ 86) ging dem Ringschluß zum Lactam (Stufe 86 $\rightrightarrows 87$ ) und dessen spontaner Cyclolisierung (Stufe $\overline{87}$ $\rightarrow \overline{88}$ ) voran. Wie die Existenz von Ergocristam beweist, ist die Reihenfolge der analogen Stufen in vivo gerade umgekehrt.

Immerhin zeigt die biomimetische Synthese, daß lineare Tripeptide mit aktiviertem C-terminalem LProlinende und einer am Aminoende geschützten $\alpha$ Hydroxy- $\alpha$-aminocarbonsäure, wie ein Beispiel in Verbindung 86 vorliegt, unter physiologischen Bedingungen zu den entsprechenden tricyclischen, geschützten Peptidteilen der Ergopeptine cyclisieren, wie ein solcher beispielsweise in Verbindung 88 vorliegt. Wenn das auch für die Biogenese-Hypothese noch keinen eindeutigen Beweis darstellt, so zeigt die chemische Synthese doch zumindest die reale Möglichkeit des dargelegten Reaktionsverlaufes auf.

\section{Zur Mikrobiologie der Ergotalkaloide}

In ähnlicher Weise, wie die Ergot-BiosyntheseForschung Erkenntnisse aus der Entdeckung neuer Alkaloide gewonnen hat, so hat auch die Ergot-Mikrobiologie-Forschung zumindest teilweise auf den Ergebnissen der Biosynthese aufbauen und so zu neuen Resultaten kommen können. Über den jeweiligen Stand der Ergot-Mikrobiologie referieren zusammenfassende Arbeiten [56, 57].

Nach den bahnbrechenden Erfolgen mit Submerskulturen von zwei Stämmen von C. paspali Stevens et HALL, von denen der eine d-Lysergsäurehydroxyäthylamid und d-Lysergsäureamid [58], der andere Paspalsäure, d-Lysergsäure und d-Isolysergsäure produziert [49], dauerte es noch Jahre, bis Stämme von C. purpurea zu effizienter Produktion von Ergopeptinen in Submerskultur weiterentwickelt werden konnten [57].

Die Schwierigkeiten, die dabei, weitgehend empirisch, überwunden werden mußten, waren von mannigfacher Art: Eine solche Fermentation ist ein ungemein komplexer Prozeß, bei dem zahlreiche biotechnologische, physiologische und genetische Faktoren zusammenspielen. In der Dynamik des Geschehens wechseln zahlreiche Parameter stündlich, ein Abweichen vom Optimum vermindert die Ausbeuten rasch. Zu optimierende, variable Parameter sind: Zusammensetzung und Konzentration des Nährmediums, Temperatur, pH, Sauerstoffpartialdruck, um nur die wichtigsten zu nennen. Wegen der langen Dauer des Fermentationsprozesses von über 14 Tagen ist eine extrem gute Sterilisation Voraussetzung. $C$. purpurea ist gegen mechanischen Stre $B$ und lokalen Sauerstoffmangel sehr empfindlich und neigt auBerdem stark zur Degeneration. Die alkaloidproduzierenden Stämme bestehen aus purpurfarbigen bis violetten, dickwandigen und isodiametrischen Zellen, welche bei der irreversiblen Degeneration in unproduktive, farblose, fadenförmige, leicht sporenbildende und stark vakuolisierte Zellen übergehen [59,
60]. Die Hauptmenge der Ergopeptine findet sich in den Mycelien, nur ein geringer Teil in der Kulturflüssigkeit, so daß Probleme bei der Isolierung entstehen.

Trotzdem gelang es schließlich, Claviceps-Stämme und Fermentationsbedingungen zu finden, die gestatten, Ergopeptine in technischem Maßstab und in Grammmengen pro Liter, zu produzieren: Bekannt sind Stämme für Ergotamin [61], $\alpha$-Ergosin [62], Ergotamin und Ergokryptin [63], Ergotoxin [64], Ergokryptin und Ergocornin [65] und auch für $\beta$-Ergokryptin [66].

Ausgangsmaterial für die Gewinnung von Produktionsstämmen waren bei uns [57] Wildstämme von Claviceps purpurea, die über Jahre hinweg in Europa und Nordamerika gesammelt worden waren. Von jedem Wildstamm wurden einige wenige Sklerotien colorimetrisch und chromatographisch auf den Alkaloidgehalt untersucht. Geeignet erscheinende Proben wurden dann unter standardisierten Bedingungen in Schüttelkolben kultiviert und die Kulturen wieder auf Alkaloidgehalt analysiert. Diejenigen Kulturen, die Alkaloide saprophytisch produzierten, wurden nun schrittweise durch das klassische Verfahren von Mutation und Auslese weiter entwickelt, wobei gleichzeitig Medienadaptionen erfolgten. Mutanten mit höherer Alkaloidproduktion konnten in der Regel durch eine Erhöhung des C- und N-Angebotes $\mathrm{zu}$ nochmals erhöhter Produktion gebracht werden. Die Mutationsprogramme wurden schrittweise durchgeführt, indem Suspensionen von Conidien einem bestimmten Mutagen ausgesetzt, die behandelten Sporen dann ausplattiert, je etwa tausend Kolonien isoliert und in Schüttelkulturen auf Alkaloidproduktion getestet wurden. Die Mutagene wurden bei jedem folgenden Schritt geändert, verwendet wurden beispielsweise der Reihe nach Äthylmethansulfonat, Äthylenimin, Nitrit und Ultraviolett.

Die so angefallenen Produktionsstämme waren weitgehend autark bezüglich der einzelnen biosynthetischen Bausteine, so daß beispielsweise die $\mathrm{Zu}$ gabe von L-Tryptophan zur Kulturflüssigkeit die Produktion der Ergopeptine nicht mehr erhöhte. Interessante Ergebnisse zeitigte jedoch die Zugabe von einzelnen Baustein-Aminosäuren an einen aus einem einzelnen Conidium gezüchteten homocaryotischen Stamm, der Ergocornin, $\alpha$ - und $\beta$-Ergokryptin nebeneinander produziert: Nach Zugabe von $5 \mathrm{~g} \mathrm{~L}$ Valin zum Liter Kulturflüssigkeit wurde $100 \%$ mehr Ergocornin, 42, gebildet, während gleichzeitig weder das Gesamtmycelgewicht noch der Gesamtalkaloidgehalt anstiegen. Die Zugabe von $5 \mathrm{~g} / \mathrm{l}$ L-Leucin, dem Precursor des $\alpha$-Ergokryptins, 38, erhöhte dessen Bildung um $50 \%$, und dies wiederum auf Kosten der beiden andern Alkaloide. Eine Konzentration von $5 \mathrm{~g}$ L-Isoleucin im Liter Kulturflüssigkeit führte schließlich zu $120 \%$ mehr $\beta$-Ergokryptin, 40 , erneut ohne Erhöhung der Gesamtalkaloidmenge [57]. 
In den letzten Jahren hat sich in der Ergot-Mikrobiologie eine neue Entwicklung angebahnt, die, wie die ersten Resultate zeigen, sicher noch weitere interessante Ergebnisse zeitigen wird: Man konfrontiert Ergopeptin-Produktionsstämme mit bausteinähnlichen, aber abiogenen Aminosäuren und untersucht, ob, wie und in welchem Ausmaß die fremde Aminosäure in das Ergopeptin-Gerüst eingebaut wird. Auf diese Weise kann einmal die Spezifizität des ergopeptinbildenden Enzymsystems abgeklärt werden, gleichzeitig fallen neue Verbindungen von medizinalchemischem Interesse an, die sonst nur in einer vielstufigen Synthese hergestellt werden könnten.

Bei der Inkubation des Ergotamin produzierenden C. purpurea Stammes PCCE 1 mit 9,10-Dihydrolysergsäure wurde das entstandene Ergotamin von $12 \% 9,10$-Dihydroergotamin, 2, begleitet [67], 9,10Dihydrolysergsäure ist also vom Pilz teilweise, anstelle von d-Lysergsäure, als Baustein zur Ergopeptinbiosynthese herangezogen worden. Der Befund ist bemerkenswert, selbst unter Berücksichtigung der Tatsache, daß 9, 10-Dihydro- $\alpha$-ergosin seit längerer Zeit in den Sklerotien von Sphacelia sorghi Mc

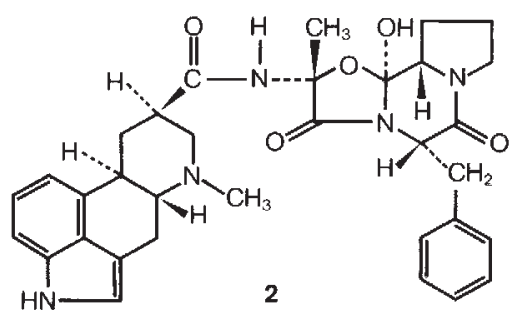

9,10-DIHYDROERGOTAMIN

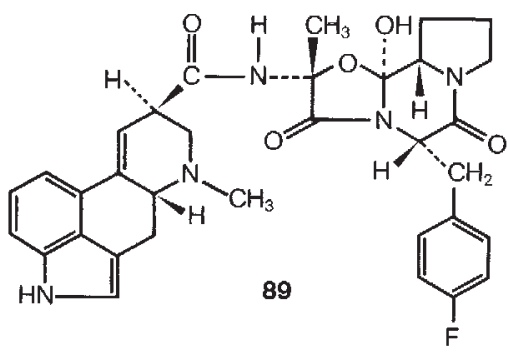

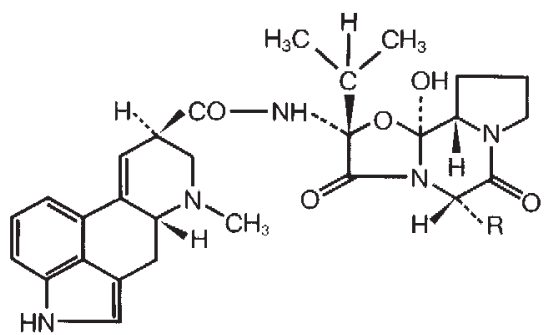

$92: \mathrm{R}=\mathrm{C}_{2} \mathrm{H}_{5}$

93: $\mathrm{R}=\mathrm{CH}_{2} \cdot \mathrm{CH}_{2} \cdot \mathrm{CH}_{3}$

$94: \mathrm{R}=\mathrm{CH}_{2} \cdot \mathrm{CH}_{2} \cdot \mathrm{CH}_{2} \cdot \mathrm{CH}_{3}$

$95: \mathrm{R}=\mathrm{CH}_{2} \cdot \mathrm{CH}_{2} \cdot \mathrm{CH}_{2} \cdot \mathrm{CF}_{3}$

$96: \mathrm{R}=\mathrm{CHOH} \cdot \mathrm{CH}_{2} \cdot \mathrm{CH}_{2} \cdot \mathrm{CH}_{3}$

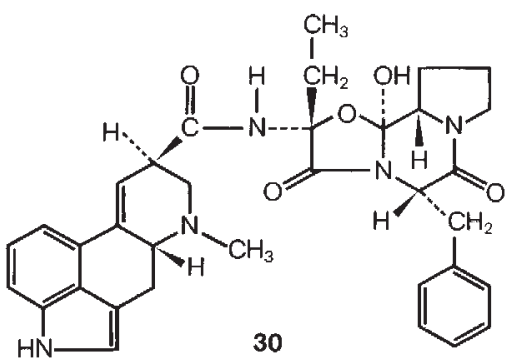

ERGOSTIN

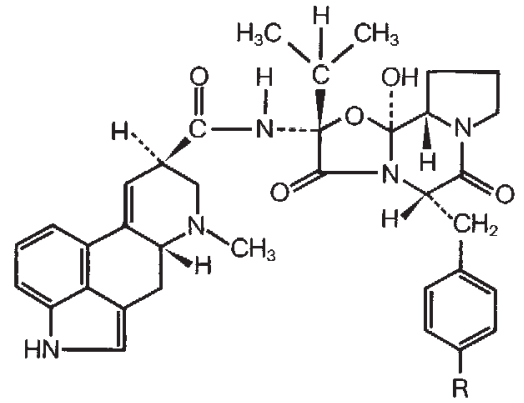

$90: R=F \quad 91: R=C l$

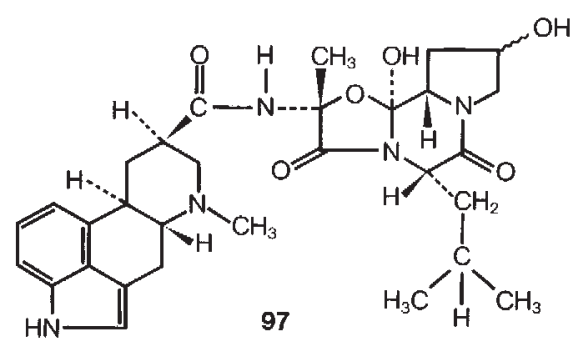

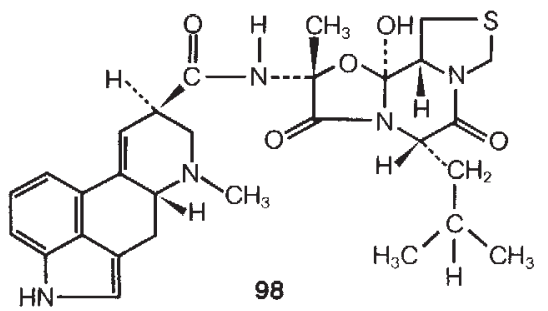

Fig. 10. Ergopeptinderivate aus mikrobiologischen Synthesen. 
RAE nachgewiesen ist [68]. Der Pilz parasitiert auf Sorghum vulgare Pers. und ist leicht zu züchten [69]. Die Biosynthese von 9,10-Dihydro- $\alpha$-ergosin verläuft aber in wichtigen Stufen anders als die der Ergopeptine [70].

Der Stamm C. purpurea 275 F.I. produziert Ergotamin in Submerskultur als Hauptalkaloid, aber kein Ergostin. Nach Zugabe von radioaktiv markierter $\alpha$ Amino-buttersäure in das Kulturmedium fand sich im Rohalkaloidgemisch $13 \%$ radioaktives Ergostin, 30, während gleichzeitig der Ergotamingehalt von $\overline{79} \%$ auf $65 \%$ absank [71]. Fast die gesamte, nicht in das Ergostin eingebaute Radioaktivität verblieb in der unveränderten $\alpha$-Amino-buttersäure. Das Resultat zeigt, daß die in der Natur selten anzutreffende $\alpha$-Amino-buttersäure L-Alanin als Baustein für die $\alpha$-Hydroxy- $\alpha$-aminosäure zu ersetzen vermag.

Da ja die zweite Aminosäure des Ergopeptingerüstes, verantwortlich für die Art des Substituenten in Stellung 5', schon eine gewisse natürliche Variationsbreite aufweist, verwundert es nicht, daß hier die künstlichen Substitutionsmöglichkeiten besonders groß sind [72]. So synthetisierte der Ergotaminstamm $C$. purpurea 275 F.I. bei Zugabe von p-Fluoro-phenylalanin das $2 \beta-$ Methyl-5' $\alpha$-(p-fluorobenzyl)-ergopeptin, 89 , mit $30 \%$ des Rohalkaloidgemisches. Sogar doppelt so hoch waren die Ausbeuten an den nah verwandten Verbindungen 90 beziehungsweise 91 bei der Alkaloidproduktion des phenylalanin-abhängigen Ergocristinstammes S 40 phe in Gegenwart von p-Fluoro-phenylalanin beziehungsweise von p-Chloro-phenylalanin. Der Ergokryptin/Ergocorninstamm B 21 erwies sich als extrem anpaßungsfähig, er baute $\alpha$-Amino-buttersäure, L-Nor-valin, L-Nor-leucin, 5,5,5-Trifluoro-leucin und $\beta$-Hydroxy-leucin ein, wobei die Verbindungen 92 bis 96 resultierten. Verbindung 92 ist nach allerneuesten Angaben ein natürliches Alkaloid; es wurde Ergobutyrin genannt und wird neben Ergobutin, dem entsprechenden Alkaloid der Ergoxingruppe, in sehr kleinen Mengen durch $C$. purpurea 231 F.I. gebildet [75].

Erstaunlicherweise gelang es in Spezialfällen, chemisch nah verwandte Verbindungen anstelle von LProlin, dem dritten Aminosäure-Baustein aller natürlichen Ergopeptine, einbauen zu lassen. So bauten Kulturen von Sphacelia sorghi bei der Inkubation mit allo-4-Hydroxy-prolin oder cis-4-Hydroxy-prolin die beiden Verbindungen zu einem geringen Prozentsatz in das Gerüst des 9,10-Dihydro- $\alpha$-ergosins ein, wobei die beiden epimeren 9 '-Hydroxydihydroergosine der Formel 97 entstanden [73]. Der $\alpha$-Ergosinstamm C. purpurea MUT 168/2 war sogar imstande, L-Thiazolidin-4-carbonsäure anstelle von LProlin zu verwerten und damit $2^{\prime} \beta$-Methyl-5' $\alpha$-isobutyl-9'-thiaergopeptin, 98, aufzubauen [74].

Zusammenfassend kann ausgesagt werden, daß im Ergopeptinmolekül alle vier Bausteine in gewissen
Grenzen austauschbar sind, die Spezifizität der synthetisierenden Enzymkombination also nicht sehr ausgeprägt ist.

\section{Literatur}

(1) Stoll, A.: Schweiz. Pat. Nr. 79879 (1918)

(2) Dudley, H. W. und C. Moir:

Brit. med. J. I, 520 (1935)

Stoll, A. und E. Burckhardt: C. R. Acad. Sci. Paris 200, 1680 (1935).

Kharash, M. S. und R. R. Legault: Science $81,388,614(1935)$.

Thomson, M. R.: Science 81, 636 (1935).

(3) Stoll, A. und A. Hofmann: Hoppe-Seyler's Z. physiol. Chem 251, 155 (1938)

(4) Stoll, A. und A. Hofmann: Helv. chim. Acta 26, 944 (1943).

(5) Stoll, A. und A. Hofmann:

Helv. chim. Acta 26, 2070 (1943).

(6) Troxler, F. und A. Hofmann: Helv. chim. Acta 40, 1721 (1957).

(7) Schneider, H. R., P. A. Stadler, P. Stütz, F. Troxler und J. Se res: Experientia 33, 1412 (1977).

(8) Floss, H. G.: Tetrahedron 32 873 (1976).

Floss, H. G., J. A. Anderson in J. D. Bu'Lock (Ed.): The Biosynthesis of Mycotoxins, p. 17, New York und London 1980, Academic Press (9) Horwell, D. C. und J. P. Verge: Phytochemistry 18,519 (1979). (10) Maier, W., D. Erge, J Schmidt und D. Gröger: Experientia 36,1553 (1980).

Maier, W., D. Erge und D. Gröger: Planta medica 40, 104 (1980). (11) Naidoo, B., J. M. Cassady, G. E. Blair und H. G. Floss: Chem. Commun. 471 (1970).

Floss, H. G., M. Tscheng-Lin, Ch.J. Chang, B. Naidoo, G. E. Blair, Ch. I. Abou-Chaar und J. M. Cassady: J, Am. chem. Soc. 96, 1898 (1974)

(12) Choong, T.: Dissertation University of Utah, Salt Lake City, Utah, USA (1978).

(13) Stütz, P., R. Brunner und P. A. Stadler: Experientia 29, 936 (1973).

(14) Flieger, M., M. Wurst, J. Stuchlik und Z. Rehacek: J. Chromatogr. 207, 139 (1981).

(15) Rutschmann, J. und P. A Stadler in: B. Berde und $\mathrm{H} . \mathrm{O}$ Schild, (Eds.) Ergotalkaloids and Related Compounds, p. 33, Berlin, Heidelberg, New York 1978, Springer.

(16) Brunner, R., P. L. Stütz, H. Tscherter und P. A. Stadler: $\mathrm{Ca}$ nad. J. Chem. 57, 1638 (1979).

(17) Schlientz, W., R. Brunner, P. A. Stadler, A. J. Frey, H. Ott und A. Hofmann: Helv. chim. Acta 47, 1921 (1964).

(18) Stütz, P., P. A. Stadler und A Hofmann: Helv. chim. Acta 53 , 1278 (1970).

(19) Porter, J. K., C. W. Bacon, J. D. Robbins und D. Betowski: J Agric. Food Chem. 29, 653 (1981).
(20) Krajicek, A., B. Trtik, J. SpaRehacek: Coll. Czeck. Chem. Commun. 44, 2255 (1979)

(21) Tyler, V. E.: J. Pharm. Sci. 50,629 (1961).

Winkler, K. und G. Gröger: Pharmazie 17,658 (1962).

Weygand, F. und H. G. Floss: Angew. Chem. Intern. Ed. 2, 243 (1963).

Voigt, R.: Pharmazie 23, 285, 353, 419 (1968).

Gröger D. in: K. Mothes, H. R. Schütte (Eds.) Biosynthese der Alkaloide p. 486, Berlin (1969), Deut. Verlag Wiss.

Floss, H. G.: Abh. Dt. Akad. Wiss., Berlin 395 (1971).

Thomas, R. und R. A. Bassett: Progr. Phytochem. 3, 47 (1972).

(22) Mothes, K., F. Weygand, D Gröger und H. Grisebach: Z. Naturforsch. 13b, 41 (1958).

Gröger, Dr., H. J. Wendt, K. Mothes und F. Weygand: Z. Naturforsch. $14 b, 355$ (1959)

Taber, W. A. und L. C. Vining Chem. Ind. 1218 (1959)

Plieninger, H., R. Fischer, W Lwowski, A. Brack, H. Kobel und A. Hofmann: Angew. Chem. 71 , 383 (1959).

Baxter, R. M., S. I. Kandel und A Okany: Chem. Ind. 266 (1960) (23) Gröger, D., K. Mothes, H. Simon, H. G. Floss und F. Weygand: Z. Naturforsch. $15 b, 141$ (1960).

(24) Birch, A. J., B. J. McLoughlin und $H$. Smith: Tetrahedron Lett. No. 7, 1 (1960).

Taylor, E. H. und E. Ramstad: Nature 188, 494 (1960), J. Pharm. Sci. $50,681(1961)$

Baxter, R. M., S. I. Kandel und A. Okany: Tetrahedron Lett. 596 (1961).

Plieninger, H., H. Immel und A. Völkl: Liebig's Ann. Chem. 706 223 (1967).

Bhattacharji, S., A. J. Birch, A Brack, A. Hofmann, H. Kobel, D C. C. Smith, H. Smith und J. Winter: J. Chem. Soc. 421 (1962).

(25) Baxter, R. M., S. I. Kandel und A. Okany: Chem. Ind. 1453 (1961).

Baxter, R. M., S. I. Kandel, A. Okany und R. G. Pyke: Canad. J. Chem. 42, 2936 (1964).

(26) Robbers, J. E. und H. G Floss: Arch. Biochem. Biophys. 126, 967 (1968)

(27) Agurell, S. und J.-E. Lindgren: Tetrahedron Lett. 5127 (1968)

(28) Agurell, S.: Acta Pharm. Suecica 3,11 (1966)

(29) Stauffacher, D. und $H$. Tscherter: Helv. chim. Acta 47, 2186 (1964). cil, P. Sedmera, J. Vokoun und Z. 
(30) Gröger, D., D. Erge und $H$. G. Floss: Z. Naturforschg. $21 b, 827$ (1966)

(31) Fehr, T., W. Acklin und D Arigoni: Chem. Commun, 801 (1966)

(32) Floss, H. G., U. Hornemann, N. Schilling, D. Gröger und D. Erge: Chem. Commun 105 (1967).

(33) Voigt, R., M. Bornschein und G. Rabitzsch: Pharmazie 22, 326 (1967).

(34) Floss, H. G., U. Hornemann, N. Schilling, K. Kelley, D. Gröger und D. Erge: J. Amer. chem. Soc. $90,6500(1968)$

(35) Pachlatko, J. P.: Dissertation Nr. 5481 ETH Zürich (1975)

(36) Ogunlana, E. O., B. J. Wilson, V, E. Tyler und E. Ramstad: Chem. Commun. 775 (1970).

(37) Stoll, A., A. Brack, H, Kobel, A. Hofmann und R. Brunner: Helv chim. Acta 37, 1815 (1954).

(38) Agurell, S. und E. Ramstad: Tetrahedron Lett. 501 (1961).

(39) Mothes, K., K. Winkler, D Gröger, H. G. Floss, U. Mothe und $F$. Weygand: Tetrahedron Lett. $933(1962)$.

(40) Kobel, H., E. Schreier und J. Rutschmann: Helv. chim. Acta 47, 1052 (1964).

(41) Ohashi, T., S. Aoki und M. Abe: J. Agr. Chem. Soc. 44, 527 (1970).

Agurell, S.: Acta Pharm. Suecica 3 65 (1966).

(42) Stoll, A. und E. Burckhardt Hoppe-Seyler's Ztschr. f. physiol Chem. 250, 1 (1937).
(43) Minghetti, A. und F. Arcamone: Experientia 25, 926 (1969).

(44) Maier, W., D. Erge und G. Gröger: Biochem. Physiol. Pflanzen (DDR) 161,559 (1971).

(45) Floss, H. G., G. P. Basmadjian, M. Tcheng, D. Gröger und D. Erge: Lloydia 34, 446 (1971).

(46) Gröger, D. und D. Erge: Z. Naturforschg. 25b, 196 (1970).

(47) Bassett, R. A., E. B. Chain und K. Corbett: Biochem. J. 134, 1 (1973)

(48) Vining, L. C. und W. A. Taber: Can. J. Microbiol. 9, 291 (1963).

(49) Agurell, S.: Acta pharm. Suecica 3, 71 (1966).

(50) Ramstad, E.: Lloydia 31, 327 (1968).

(51) Floss, H. G., M. TchengLing, H. Kobel und P. A. Stadler: Experientia 30, 1369 (1974).

(52) Stadler, P. A.: Kem. Ind. 29 , 207 (1980).

(53) Lucente, G., G. Fiorentini und D. Rossi: Gazz. Chim. Ital 101, 109 (1971).

Lucente, G. und A. Romeo: Chem. Commun. 1605 (1971).

Lucente, G. und P. Frattesi: Tetrahedron Lett. 4283 (1972).

Lucente, G., A. Romeo und G. Zanotti: Gazz. Chim. Ital. 102, 941 (1972).

(54) Stütz, P. und P. A. Stadler: Monatshefte Chemie 107,763 (1976).

(55) Hofmann, A., H. Ott, R. Griot, P. A. Stadler und A. J. Frey: Helv. chim. Acta 46, 2306 (1963).
(56) Bove, F. J.: The Story of Ergot, Basel und New York 1970, Karger.

Gröger, D., in: Kadis, S., A. Ciegler und S. J. Ajl (Eds.) Microbial Toxins 8, 321, New York und London 1972, Academic Press.

Mantle, P. G., in: Smith, J. E. und D. R. Berry (Eds.) The Filamentous Fungi 1,281 , London 1975

Edward Arnold.

(57) Kobel, H., J. J. Sanglier in: R. Hütter (Eds.) Antibiotics and $\mathrm{Ot}-$ her Secondary Metabolites, Biosynthesis and Production, FEMS Symposium No. 5, 233, New York und London 1978, Academic Press. (58) Arcamone, F., C. Bonino, E B. Chain, A. Ferretti, P. Penella, A. Tonolo und L. Vero: Nature $187,238(1960)$.

(59) Mantle, P. G. und A. Tonolo: Trans. Br. Mycol. Soc. 5I, 499 (1968).

(60) Kobel, H.: Pathologia et Microbiologia 34, 249 (1969).

(61) Spalla, C. und L. Tognoli: Experientia 22, 415 (1966).

(62) Gröger, D., H. P. Schmauder, S. Johne, W. Maier und D. Erge: DDR-Patent 129801 (1977)

(63) Amici, A. M., A. Minghetti, T. Scotti und C. Spalla: Deutsche Auslegeschrift 1792038 (1972). (64) Kobel, H. und J. J. Sanglier: Schweizerisches Patent 577556 (1975).
(65) Wack, G., L. Nagy, D. Szekely, J. Szolnoky, E. Udvardy-Nagy und E. Zsoka: Deutsche Offenlegungsschrift 2336765 (1974).

(66) Bianchi, M., A. Minghetti und C. Spalla: Experientia 32, 145 (1976).

67) Anderson, J. A., I.-S. Kim, P. Lehtonen und H. G. Floss: J. Nat. Prod. 42, 271 (1979).

(68) Mantle, P. G.: Annals of Applied Biology 62, 443 (1968).

Mantle, P. G. und E. S. Waight: Nature 218, 581 (1968)

(69) Mantle, P. G.: J. Gen. Microbiology 75, 275 (1973).

(70) Barrow, K. D., P. G. Mantle und F, R. Quigley: Tetrahedron Lett. 1557 (1974)

(71) Crespi-Perellino, N., A. Guicciardi, A. Minghetti und C. Spalla: Experientia 37, 217 (1981).

(72) Beacco, E., M. L. Bianchi, A. Minghetti und C. Spalla: Experientia 34, 1291 (1978); Deutsche Offenlegungsschrift 2816773 (1978). (73) Atwell, S. M. und P. G. Mantle: Experienta 37, 1257 (1981)

(74) Baumert, A., D. Erge und D. Gröger: Planta medica 43,122 (1982).

(75) Bianchi, M. L., N. Crespi-Perellino, B. Gioia und A. Minghetti: J. Natural Prod. 45, 191 (1982).

Adresse: Dr. P. A. Stadler, Sandoz $A G$,

Pharmazeutisches Department, Präklinische Forschung, CH-4002 Basel, Schweiz 